\title{
On the coupling of the self-dual field to dynamical U(1) Matter and its dual theory
}

\author{
D. Dalmazi \\ UNESP - Campus de Guaratinguetá - DFQ \\ Av. Dr. Ariberto Pereira da Cunha, 333 \\ CEP 12516-410 - Guaratinguetá - SP - Brazil. \\ E-mail: dalmazi@feg.unesp.br
}

January 4, 2019

\begin{abstract}
We consider an arbitrary $U(1)$ charged matter non-minimally coupled to the self-dual field in $d=2+1$. The coupling includes a linear and a rather general quadratic term in the self-dual field. By using both a Lagragian gauge embedding and a master action approaches we derive the dual Maxwell Chern-Simons type model and show the classical equivalence between the two theories. At quantum level the master action approach in general requires the addition of an awkward extra term to the Maxwell Chern-Simons type theory. Only in the case of a linear coupling in the self-dual field the extra term can be dropped and we are able to establish the quantum equivalence of gauge invariant correlation functions in both theories.
\end{abstract}

PACS-No.: 11.15.-q, 11.10.Kk, 11.10.Gh, 11.10.Ef 


\section{Introduction}

It is very common in physics that due to technical difficulties we are not able to probe, even at theoretical level, all physical aspects of a given theory. In those cases it is important to have an equivalent ( dual ) description of the same theory. A classical example is the dual description of the massive Thirring model in $d=1+1$ by means of the massive SineGordon model [1, 2] (see also [3] and references therein). The strong coupling expansion in one model corresponds to the usual weak coupling expansion in the other one and viceversa. Some nonperturbative features like confinement can also be revealed with the help of duality as in 44. In this work we are interested in the specific case of dual descriptions of a spin one massive particle in $d=2+1$ (see [5, 6]). This type of duality naturally appears in the bosonization program in $d=2+1$ where fermionic $U(1)$ current correlators in the massive Thirring model can be equally described by a bosonic spin one massive field which, for infinitely massive fermions, may be either of self-dual (SD) or Maxwell Chern-Simons (MCS) type [7, 8, 9, 10, 11, 12].

By means of a master action approach it was shown in [6] that the gauge invariant sector of the MCS theory is on shell equivalent to the SD theory with the dual map $f_{\mu} \leftrightarrow$ $\frac{\epsilon_{\mu \nu \alpha} \partial^{\nu} A^{\alpha}}{m}$. The equivalence holds beyond the classical level and includes gauge invariant correlation functions in the quantum theory as shown in [13. Although the equivalence between those free theories is interesting in its own, the most powerful applications of duality occur in interacting theories. The case of the non-abelian version of a possible $\mathrm{SD} / \mathrm{MCS}$ duality is much less trivial and it is still under investigation [14. Another possibility to build an interacting theory is to couple the vector fields in SD and MCS theories to $U(1)$ charged matter fields. Along this direction it has been shown in [15] that the SD field minimally coupled to $U(1)$ charged massive fermions is dual equivalent to a MCS gauge field coupled to matter through a Pauli term. Besides, a Thirring term must be added to assure the equivalence also in the fermionic sector. The demonstration of [15, which is based on a master action, guarantees the equivalence of the equations of motion as well as of the partition functions of the dual theories. More recently, in [16] both cases of $U(1)$ charged fermions and scalar fields minimally coupled to the SD field have been considered through a Lagrangian gauge embedding procedure. In particular, the results of [15] have been reproduced at classical level pointing out that the dual map must include now a contribution from the matter fields as we will see later in this work. The case of scalar fields minimally coupled to the SD field leads to a MCS theory in a field dependent medium, a highly nonlinear theory. Once again, the minimal coupling is replaced by a Pauli term in the dual MCS type theory and a current-current term 
must be introduced to guarantee equivalence in the scalar sector. Similar applications of the Lagrangian gauge embedding procedure to produce gauge theories dual to non-gauge theories have been worked out, for instance in [17, 18]. Only the classical equivalence has been considered in [16, 17, 18. In those cases the matter fields are always minimally coupled to the vector field. Since those vector fields are not gauge fields there is no reason a priori to use minimal coupling. In other words, it is not clear why should one couple those fields to a conserved current, as already remarked in [6]. The aim of this work is to clarify this issue in the literature by assuming a rather general coupling between the selfdual field and an arbitrary $U(1)$ charged matter. In section 2 we present a simple proof of on shell equivalence between the corresponding SD and MCS theories non-minimally coupled to dynamical $U(1)$ matter. The proof is independent of the details of the matter theory. In section 3 we analyze the equivalence of gauge invariant correlation functions in the quantum theory and draw some conclusions in section 4.

\section{On shell equivalence}

\subsection{Lagrangian gauge embedding}

We start from the Lagrangian of the self-dual field coupled to some $U(1)$ charged matter fields. Due to the mass term in the SD theory we have no gauge invariance and therefore we are not constrained to use the minimal coupling condition. The coupling will be rather general and will contain a linear and a quadratic term in the self-dual field such that the global $U(1)$ symmetry of the matter theory is preserved, namely

$$
\mathcal{L}_{M+S D}=\mathcal{L}_{S D}+\frac{U\left(\varphi, \varphi^{*}\right)}{2} f^{\nu} f_{\nu}-e f^{\nu} J_{\nu}^{(0)}+\mathcal{L}_{\text {Matter }}^{U(1)}
$$

Where we define:

$$
\begin{aligned}
\mathcal{L}_{S D} & =\frac{m^{2}}{2} f^{\mu} f_{\mu}-\frac{m}{2} \epsilon_{\alpha \beta \gamma} f^{\alpha} \partial^{\beta} f^{\gamma} \\
\mathcal{L}_{\text {Matter }}^{U(1)} & =\mathcal{L}_{\text {Kinetic }}+V\left(\varphi, \varphi^{*}\right)
\end{aligned}
$$

The generic notation $\left(\varphi, \varphi^{*}\right)$ represents the matter fields which may be either fermions, scalars or even complex vector fields. The function of the matter fields $U\left(\varphi, \varphi^{*}\right)$ is positive and global $U(1)$ invariant, but otherwise arbitrary. Likewise, $V\left(\varphi, \varphi^{*}\right)$ is also assumed to be an arbitrary and global $U(1)$ invariant function which may include a mass term while $\mathcal{L}_{\text {Kinetic }}$ represents the purely kinetic terms. The current $J_{\nu}^{(0)}$ is the $U(1)$ Noether current 
which comes from the free matter theory $\mathcal{L}_{\text {Kinetic }}$. Minimizing the action we obtain the equations of motion :

$$
\begin{gathered}
K_{\nu} \equiv \frac{\delta S}{\delta f^{\nu}}=\left(\mu^{2} g_{\nu \alpha}+m \epsilon_{\nu \alpha \gamma} \partial^{\gamma}\right) f^{\alpha}-e J_{\nu}^{(0)}=0 \\
\int d^{3} x\left(\delta \mathcal{L}_{\text {Matter }}^{U(1)}-e f^{\nu} \delta J_{\nu}^{(0)}+\frac{\delta \mu^{2}}{2} f^{\nu} f_{\nu}\right)=0
\end{gathered}
$$

Where we use the notation:

$$
\mu^{2}=m^{2}+U\left(\varphi, \varphi^{*}\right)
$$

Dropping the matter fields, it is known $[5,6]$ that $\mathcal{L}_{S D}$ is dual to the Lagrangian

$$
\mathcal{L}_{M C S}=\frac{m}{2} \epsilon_{\alpha \beta \gamma} A^{\alpha} \partial^{\beta} A^{\gamma}-\frac{1}{4} F_{\mu \nu}(A) F^{\mu \nu}(A) .
$$

Therefore, one might ask what is the Lagrangian dual to $\mathcal{L}_{M+S D}$. The basic idea is to add extra terms to (11) such that the new theory is invariant under the gauge transformation $\delta f_{\nu}=\partial_{\nu} \alpha ; \delta \varphi=0$ while keeping its equivalence to the old theory. Adapting the procedure of [16] to our case leads to :

$$
\mathcal{L}_{M+M C S}=\mathcal{L}_{M+S D}-\frac{1}{2 \mu^{2}} K^{\nu} K_{\nu}
$$

It is a simple exercise to check that $\mathcal{L}_{M+M C S}$ is invariant under the aforementioned gauge transformations. Using $\delta K_{\nu}=\mu^{2} \delta f_{\nu}+\delta \mu^{2} f_{\nu}-e \delta J_{\nu}^{(0)}+\epsilon_{\nu \alpha \gamma} \partial^{\gamma} \delta f^{\alpha}$, after an integration by parts and a trivial cancellation, we can write down the equations of motion of $\mathcal{L}_{M+M C S}$ in the form :

$$
\begin{gathered}
\epsilon^{\nu \gamma \alpha} \partial_{\alpha}\left(\frac{K_{\gamma}}{\mu^{2}}\right)=0 \\
\int d^{3} x\left[\delta \mathcal{L}_{\text {Matter }}^{U(1)}-e\left(f^{\nu}-\frac{K^{\nu}}{\mu^{2}}\right) \delta J_{\nu}^{(0)}+\frac{\delta \mu^{2}}{2}\left(f^{\nu}-\frac{K^{\nu}}{\mu^{2}}\right)^{2}\right]=0 .
\end{gathered}
$$

From the definition of $K^{\nu}$ in (44) we have the identity

$$
-m \epsilon_{\nu \alpha \gamma} \partial^{\gamma}\left(\frac{K^{\alpha}}{\mu^{2}}\right)=\left(\mu^{2} g_{\nu \alpha}+m \epsilon_{\nu \alpha \gamma} \partial^{\gamma}\right)\left(f^{\alpha}-\frac{K^{\alpha}}{\mu^{2}}\right)-e J_{\nu}^{(0)}
$$

Thus, (9) and (10) are equivalent to

$$
\left(\mu^{2} g_{\nu \alpha}+m \epsilon_{\nu \alpha \gamma} \partial^{\gamma}\right) \tilde{f}^{\alpha}-e J_{\nu}^{(0)}=0
$$




$$
\int d^{3} x\left(\delta \mathcal{L}_{\text {Matter }}^{U(1)}-e \tilde{f}^{\nu} \delta J_{\nu}^{(0)}+\frac{\delta \mu^{2}}{2} \tilde{f}^{\nu} \tilde{f}_{\nu}\right)=0
$$

which have the same form of the equations of motion of $\mathcal{L}_{M+S D}$, see (44) and (5), with $f_{\nu}$ being replaced by :

$$
\tilde{f}_{\nu}=f_{\nu}-\frac{K_{\nu}}{\mu^{2}}=-\frac{m}{\mu^{2}} \epsilon_{\nu \alpha \gamma} \partial^{\gamma} f^{\alpha}+\frac{e}{\mu^{2}} J_{\nu}^{(0)}
$$

The equations (12) and (13) are gauge invariant as a consequence of the gauge invariance of $\tilde{f}_{\nu}$. Solving (12) for $\tilde{f}_{\nu}=\tilde{f}_{\nu}\left(\varphi, \varphi^{*}\right)$ and plugging back in (13) will furnish equations of motion involving only matter fields which are, of course, the same equations obtained by solving (41) for $f_{\nu}=f_{\nu}\left(\varphi, \varphi^{*}\right)$ and substituting back in (5). That proves the on shell equivalence of $\mathcal{L}_{M+M C S}$ and $\mathcal{L}_{M+S D}$ in the matter sector. Concerning the equations of motion for $f_{\alpha}$, notice that this is a gauge field in $\mathcal{L}_{M+M C S}$ and its equation of motion will depend in general on some arbitrary space-time function. We can make this arbitrariness explicit by noticing that the most general solution to (91) is:

$$
K_{\nu}=\mu^{2} \partial_{\nu} \alpha=\mu^{2}\left(g_{\nu \beta}+m \epsilon_{\nu \beta \gamma}\right) f^{\beta}-e J_{\nu}^{(0)}
$$

where $\alpha\left(x_{\mu}\right)$ is an arbitrary space-time function. By choosing $\alpha$ a constant function we recover equation (4) and the on shell equivalence between $\mathcal{L}_{M+S D}$ and $\mathcal{L}_{M+M C S}$ is fully demonstrated for arbitrary coupling $U\left(\varphi, \varphi^{*}\right)$ and generic $U(1)$ matter fields with arbitrary potential $V\left(\varphi, \varphi^{*}\right)$. In particular, no requirement of minimal coupling was necessary for the demonstration. For future use we end up this subsection writing down the full MCS type Lagrangian dual to $\mathcal{L}_{M+S D}$. From (8]) we have :

$$
\begin{aligned}
\mathcal{L}_{M+M C S}= & -\frac{m^{2}}{4 \mu^{2}} F_{\alpha \beta}(A) F^{\alpha \beta}(A)+\frac{m}{2} \epsilon_{\alpha \beta \gamma} A^{\alpha} \partial^{\beta} A^{\gamma} \\
& -\frac{m e}{\mu^{2}} J_{\nu}^{(0)} \epsilon^{\nu \alpha \beta} \partial_{\alpha} A_{\beta}-\frac{e^{2}}{2 \mu^{2}} J_{\nu}^{(0)} J^{\nu(0)}+\mathcal{L}_{\text {matter }}^{U(1)}
\end{aligned}
$$

As usual, we have renamed $f_{\nu} \rightarrow A_{\nu}$. The above highly nonlinear Lagrangian is gauge invariant $\left(\delta A_{\nu}=\partial_{\nu} \alpha\right)$ and contains a Pauli and a Thirring like term as in the simpler case of minimal coupling [16].

\subsection{Master Action}

The classical equivalence between the free theories $\mathcal{L}_{S D}$ and $\mathcal{L}_{M C S}$ was proved in [6] using a master action approach. This was later generalized in [15] for the coupling (minimal) with fermions. Here we suggest a new master action which interpolates between $\mathcal{L}_{M+S D}$ and $\mathcal{L}_{M+M C S}$ for arbitrary $U(1)$ matter. This approach is specially useful when comparing correlation functions in the quantum theory [13, 19]. 
First of all, notice that the first three terms in (16) can be combined such that

$$
\mathcal{L}_{M+M C S}=-\frac{\mu^{2}}{2}\left(\frac{m \epsilon_{\nu \alpha \beta} \partial^{\beta} A^{\alpha}-e J_{\nu}^{(0)}}{\mu^{2}}\right)^{2}+\frac{m}{2} \epsilon_{\alpha \beta \gamma} A^{\alpha} \partial^{\beta} A^{\gamma}+\mathcal{L}_{\text {matter }}^{U(1)}
$$

This formula suggests the gauge invariant master Lagrangian:

$$
\begin{aligned}
\mathcal{L}_{\text {master }} & =\frac{\mu^{2}}{2} f^{\nu} f_{\nu}+f^{\nu}\left(m \epsilon_{\nu \alpha \beta} \partial^{\beta} A^{\alpha}-e J_{\nu}^{(0)}\right) \\
& +\frac{m}{2} \epsilon_{\alpha \beta \gamma} A^{\alpha} \partial^{\beta} A^{\gamma}+\mathcal{L}_{\text {matter }}^{U(1)}
\end{aligned}
$$

where $\mu^{2}$ is defined as in (66) and $f_{\nu}$ is so far an extra non-dynamical auxiliary field. It is easy to show that the equations of motion from $\mathcal{L}_{\text {master }}$ are equivalent to the equations of motion that we get from $\mathcal{L}_{M+M C S}$ or $\mathcal{L}_{M+S D}$. Indeed, minimizing $\mathcal{L}_{\text {master }}$ we have:

$$
\begin{gathered}
\epsilon^{\gamma \beta \alpha} \partial_{\beta}\left(A_{\alpha}-f_{\alpha}\right)=0 \\
\mu^{2} f_{\nu}=e J_{\nu}^{(0)}-m \epsilon_{\nu \gamma \delta} \partial^{\delta} A^{\gamma} \\
\int d^{3} x\left(\delta \mathcal{L}_{\text {Matter }}^{U(1)}-e f^{\nu} \delta J_{\nu}^{(0)}+\frac{\delta \mu^{2}}{2} f^{\nu} f_{\nu}\right)=0 .
\end{gathered}
$$

From (19) we have $A_{\nu}=f_{\nu}+\partial_{\nu} \alpha$ with arbitrary $\alpha$. Plugging back in (20) and (21) we recover exactly the equations of motion of $\mathcal{L}_{M+S D}$. Alternatively, solving (20) for $f_{\nu}$ and returning in (21) we obtain the equations of motion of $\mathcal{L}_{M+M C S}$ in the matter sector, while returning in (19) we get exactly the equations of motion for the gauge potential coming from $\mathcal{L}_{M+M C S}$, namely,

$$
\epsilon^{\alpha \nu \beta} \partial^{\beta}\left(A_{\nu}-\frac{e J_{\nu}^{(0)}}{\mu^{2}}+\frac{m}{\mu^{2}} \epsilon_{\nu \gamma \delta} \partial^{\delta} A^{\gamma}\right)=0,
$$

That completes the proof of on shell equivalence of $\mathcal{L}_{\text {master }}$ to $\mathcal{L}_{M+M C S}$ and $\mathcal{L}_{M+S D}$. Our master action works for arbitrary $U(1)$ matter with no restrictions on $V\left(\varphi, \varphi^{*}\right)$ and $U\left(\varphi, \varphi^{*}\right)$. The minimal coupling has not played any role so far. However, there is one point which deserves to be mentioned. Namely, in the cases of minimal coupling analyzed in the literature [15, 16] and also for the free SD theory, the equation of motion for the self-dual field, see (4), leads to $\partial^{\nu} f_{\nu}=0$. This is no longer true for the arbitrary coupling $U\left(\varphi, \varphi^{*}\right)$. For instance, for scalar fields, where $J_{\nu}^{(0)}=\imath\left(\phi \partial_{\nu} \phi^{*}-\phi^{*} \partial_{\nu} \phi\right)$, we have instead

$$
\partial_{\nu}\left\{\left[m^{2}+U\left(\phi, \phi^{*}\right)-2 e^{2} \phi^{*} \phi\right] f^{\nu}\right\}=0
$$


Only in the case of minimal coupling, which corresponds to $U\left(\phi, \phi^{*}\right)=2 e^{2} \phi^{*} \phi$, we obtain the simple relation $\partial_{\nu} f^{\nu}=0$. This is quite surprising because, as we stressed before, $\mathcal{L}_{M+S D}$ is not a gauge theory and we should not expect any special results in the case of minimal coupling. Even from the point of view of the dual $M C S$ type theory this is not clear since this theory is much more complicated for $U\left(\phi, \phi^{*}\right)=2 e^{2} \phi^{*} \phi$ than it is for $U\left(\phi, \phi^{*}\right)=0$. One interesting point concerning the equation $\partial_{\nu} f^{\nu}=0$ is the counting of degrees of freedom. Since, both free theories $\mathcal{L}_{S D}$ and $\mathcal{L}_{M C S}$ represent one polarization state of a massive spin one field and $\mathcal{L}_{S D}$ is not a gauge theory like $\mathcal{L}_{M C S}$, it is necessary to have such equation to balance the counting of degrees of freedom. It plays the same role in the SD theory of a gauge condition in the MCS one. Regarding our interacting theories, it is clear that the relation (23) reduces the number of degrees of freedom of $f_{\nu}$ by the same amount whatever we choose for $U\left(\phi, \phi^{*}\right)$ as far as $U\left(\phi, \phi^{*}\right) \neq 2 e^{2} \phi^{*} \phi-m^{2}$.

1 Therefore, to the best we know, there is no mandatory reason to have the relation $\partial_{\nu} f^{\nu}=0$ in the interacting theory.

\section{Quantum equivalence}

In order to examine under which conditions the $\mathcal{L}_{M+S D} / \mathcal{L}_{M+M C S}$ duality holds at quantum level we introduce the partition function :

$$
\mathcal{Z}=\int \mathcal{D} \varphi \mathcal{D} \varphi^{*} \mathcal{D} f^{\nu} \mathcal{D} A^{\nu} e^{\imath \int d^{3} x\left[\mathcal{L}_{\text {master }}+\frac{\lambda}{2}\left(\partial^{\nu} A_{\nu}\right)^{2}\right]}
$$

Since $\mathcal{L}_{\text {master }}$ is a gauge theory we have added a gauge fixing term. The gauge field $A_{\nu}$ can be easily integrated over. Using momentum space notation we have:

$$
\begin{aligned}
& \int \mathcal{D} A^{\nu} e^{\frac{\imath}{2} \int \frac{d^{3} k}{(2 \pi)^{3}}\left[\tilde{A}^{\mu}(k) \mathcal{O}_{\mu \nu} \tilde{A}^{\nu}(-k)+\tilde{A}^{\mu}(k) T_{\mu}(-k)+\tilde{A}^{\mu}(k) T_{\mu}(k)\right]} \\
= & C \exp -\frac{\imath}{2} \int \frac{d^{3} k}{(2 \pi)^{3}} T_{\alpha}(k)\left(\mathcal{O}^{-1}\right)^{\alpha \beta} T_{\beta}(-k) .
\end{aligned}
$$

Where $C$ is a numerical factor and ${ }^{2}$

$$
\begin{aligned}
\mathcal{O}_{\alpha \beta}(k) & =\imath m E_{\alpha \beta}+\lambda k_{\alpha} k_{\beta} \\
\mathcal{O}_{\alpha \beta}^{-1}(k) & =\frac{k_{\alpha} k_{\beta}}{\lambda k^{4}}+\imath \frac{E_{\alpha \beta}}{m k^{2}}
\end{aligned}
$$

\footnotetext{
${ }^{1}$ We are not interested in that special case in this work because we would have a gauge theory governed by the Chern-Simons term instead of the self-dual theory.

${ }^{2}$ We use the same notation, without tilde, for the Fourier components $f_{\nu}(k)$ and the space-time components $f_{\nu}(x)$ to avoid confusion with the notation for the dual field (14).
} 


$$
\begin{aligned}
T_{\mu}(-k) & =-\imath m E_{\mu \alpha} f^{\alpha}(-k) \\
E_{\mu \nu} & =\epsilon_{\mu \nu \alpha} k^{\alpha} .
\end{aligned}
$$

Usually, a gaussian integral like (25) takes us from a local field theory in space-time to a nonlocal one. Remarkably, thanks to the identity $E_{\mu \nu}\left(\mathcal{O}^{-1}\right)^{\nu \alpha} E_{\alpha \beta}=(-\imath / m) E_{\mu \beta}$ we end up with a local theory which is exactly $\mathcal{L}_{M+S D}$ :

$$
\mathcal{Z}=C \int \mathcal{D} \varphi \mathcal{D} \varphi^{*} \mathcal{D} f^{\nu} e^{\imath \int d^{3} x \mathcal{L}_{M+S D}}
$$

On the other hand, starting from (24) we could have made the translation $f_{\nu} \rightarrow f_{\nu}+$ $\left(e J_{\nu}^{(0)}-m \epsilon_{\nu \alpha \beta} \partial^{\beta} A^{\alpha}\right) / \mu^{2}$ leading to the dual partition function:

$$
\mathcal{Z}=\int \mathcal{D} \varphi \mathcal{D} \varphi^{*} \mathcal{D} f^{\nu} \mathcal{D} A^{\nu} e^{\imath \int d^{3} x\left[\mathcal{L}_{M+M C S}+\frac{\lambda}{2}\left(\partial^{\nu} A_{\nu}\right)^{2}+\mathcal{L}_{\text {extra }}\right]},
$$

where $\mathcal{L}_{M+M C S}$ is given in (16) and

$$
\mathcal{L}_{\text {extra }}=\frac{m^{2}+U\left(\varphi, \varphi^{*}\right)}{2} f^{\nu} f_{\nu}
$$

At classical level, $\mathcal{L}_{\text {extra }}$ can be dropped since its only role is to produce the equation of motion $f_{\nu}=0$. This is in agreement with the classical equivalence between $\mathcal{L}_{M+S D}$ and $\mathcal{L}_{M+M C S}$ proved in the last section. At quantum level we must distinguish the general case $U\left(\varphi, \varphi^{*}\right) \neq 0$ from the case where the self-dual field appears only linearly coupled with the matter fields $U\left(\varphi, \varphi^{*}\right)=0$. In the first case we have the free propagator $\left\langle f_{\alpha}(k) f_{\beta}(-k)\right\rangle=\frac{g_{\alpha \beta}}{m^{2}}$. The vertex $\frac{1}{2} U\left(\varphi, \varphi^{*}\right) f^{\nu} f_{\nu}$ will then generate new self-interaction vertices for the matter fields after integration over $f_{\nu}$. All those new vertices have a divergent coefficient (cubic divergence) due to the bad ultraviolet behavior of the $f_{\nu}$ free propagator. In fact, the same problem can be seen from the point of view of $\mathcal{L}_{M+S D}$. There we have the free propagator of the self-dual field:

$$
\left\langle f_{\alpha}(k) f_{\beta}(-k)\right\rangle_{M+S D}=\frac{g_{\alpha \beta}}{k^{2}-m^{2}}+\frac{k_{\alpha} k_{\beta}}{m^{2}\left(k^{2}-m^{2}\right)}-\imath \frac{E_{\alpha \beta}}{m\left(k^{2}-m^{2}\right)} .
$$

As $k \rightarrow \infty$ the above propagator behaves like a constant, which is a problem already noticed in [20, 21]. Consequently the vertex $\frac{1}{2} U\left(\varphi, \varphi^{*}\right) f^{\nu} f_{\nu}$ present in $\mathcal{L}_{M+S D}$ will also lead to nonrenormalizable cubic divergences in the matter sector. Therefore it is not surprising to have such divergences in the dual MCS type theory. In fact, the bad ultraviolet behavior of the self-dual propagator is a source of troubles even for $U\left(\varphi, \varphi^{*}\right)=0$ where it leads to power counting nonrenormalizable interactions like the Thirring and the Pauli term. Hopefully, one can surmount the problems of the linear coupling case $U\left(\varphi, \varphi^{*}\right)=0$ by 
introducing extra flavors in the matter sector and using a $1 / N_{f}$ expansion. At least in the case of fermionic matter fields there are power counting arguments given in [15] ( see also [22] ) pointing to a $1 / N_{f}$ renormalizability although, a more detailed analysis and the extension to other types of matter fields is missing in the literature.

For the more general coupling $U\left(\varphi, \varphi^{*}\right) \neq 0$ the introduction of extra flavors is not enough to avoid the infinities generated by $\mathcal{L}_{\text {extra }}$ and one might try a more dramatic change in the original SD theory. It is natural to add second order derivative terms ( see similar trick in [22] ) in order to improve the ultraviolet behavior of the $f_{\nu}$ propagator. On one hand, if we introduce a gauge non-invariant term like $(\lambda / 2)\left(\partial_{\nu} f^{\nu}\right)^{2}$, although the ultraviolet behavior is certainly improved, we end up with a dual nonlocal MCS type theory. Basically we have $\mu^{2} \rightarrow \mu^{2}-\lambda \partial_{\nu} \partial^{\nu}$ and therefore $1 /\left(\mu^{2}-\lambda \partial_{\nu} \partial^{\nu}\right)$ will be the source of nonlocality in the MCS type theory. On the other hand, we can avoid nonlocality in the dual MCS theory by adding a gauge invariant extra term like $\lambda F_{\mu \nu}(f) F^{\mu \nu}(f)$ but in this case the ultraviolet behavior of $f_{\nu}$ will not be improved. In summary, there seems to be no simple solution to the infinities appearing for $U\left(\varphi, \varphi^{*}\right) \neq 0$.

At this point we simply abandon the general case and stick to $U\left(\varphi, \varphi^{*}\right)=0$ to avoid the cubic divergences. In this case the integral over $f_{\nu}$ in (311) produces only a numerical factor in the partition function :

$$
\mathcal{Z}=\tilde{C} \int \mathcal{D} \varphi \mathcal{D} \varphi^{*} \mathcal{D} A^{\nu} e^{\imath \int d^{3} x\left[\mathcal{L}_{M+M C S}+\frac{\lambda}{2}\left(\partial^{\nu} A_{\nu}\right)^{2}\right]}
$$

Thus, comparing with (30), we conclude that in the special case of linear coupling the classical equivalence between $\mathcal{L}_{M+M C S}$ and $\mathcal{L}_{M+S D}$ goes through the partition function. Likewise, we could have introduced sources for the matter fields in (24) and since no integration over the matter fields has been done so far, it would be trivial to show that the normalized matter field correlation functions must be equal in both theories:

$$
\left\langle\varphi_{a_{1}}\left(x_{1}\right) \cdots \varphi_{a_{n}}\left(x_{n}\right) \varphi_{b_{1}}^{*}\left(y_{1}\right) \cdots \varphi_{b_{n}}^{*}\left(y_{n}\right)\right\rangle_{M+M C S}^{U=0}=\left\langle\varphi_{a_{1}}\left(x_{1}\right) \cdots \varphi_{a_{n}}\left(x_{n}\right) \varphi_{b_{1}}^{*}\left(y_{1}\right) \cdots \varphi_{b_{n}}^{*}\left(y_{n}\right)\right\rangle_{M+S D}^{U=0}
$$

Regarding the vector field correlation functions, we introduce two types of sources in (24), as in the free case [13, and define the generating function:

$$
\mathcal{Z}(j, \tilde{j})=\int \mathcal{D} \varphi \mathcal{D} \varphi^{*} \mathcal{D} f^{\nu} \mathcal{D} A^{\nu} e^{\imath \int d^{3} x\left[\mathcal{L}_{\text {master }}+\frac{\lambda}{2}\left(\partial^{\nu} A_{\nu}\right)^{2}+j^{\alpha} f_{\alpha}+\tilde{j}^{\alpha} \tilde{F}_{\alpha}(A)\right]} .
$$

Where $\tilde{F}_{\nu}(A)=-(1 / m) \epsilon_{\nu \beta \gamma} \partial^{\gamma} A^{\beta}+e J_{\nu}^{(0)} / m^{2}$. After the translation $f_{\alpha} \rightarrow f_{\alpha}+\tilde{j}_{\alpha} / m^{2}$ and integration over $A_{\mu}$ we obtain : 


$$
\mathcal{Z}_{M+S D}(j, \tilde{j})=C \int \mathcal{D} \varphi \mathcal{D} \varphi^{*} \mathcal{D} f^{\nu} \mathcal{D} A^{\nu} e^{\imath \int d^{3} x\left[\mathcal{L}_{M+S D}+f_{\alpha}\left(j^{\alpha}+\tilde{j}^{\alpha}\right)+\frac{\tilde{j}^{\alpha} \tilde{j}_{\alpha}}{2 m^{2}}+\frac{j^{\alpha} \tilde{j}_{\alpha}}{m^{2}}\right]}
$$

On the other hand, starting from (36) and integrating over $f_{\nu}$ we derive

$$
\mathcal{Z}_{M+M C S}(j, \tilde{j})=\tilde{C} \int \mathcal{D} \varphi \mathcal{D} \varphi^{*} \mathcal{D} f^{\nu} \mathcal{D} A^{\nu} e^{\imath \int d^{3} x\left[\mathcal{L}_{M+M C S}+\tilde{F}_{\alpha}(A)\left(j^{\alpha}+\tilde{j}^{\alpha}\right)-\frac{j^{\alpha} j_{\alpha}}{2 m^{2}}+\frac{\lambda}{2}\left(\partial^{\nu} A_{\nu}\right)^{2}\right]}
$$

From (37) and (38) we can obtain the correlation functions. For instance, for the nonconnected two and four-point correlation functions we have:

$$
\begin{aligned}
\left\langle f_{\alpha_{1}}\left(x_{1}\right) f_{\alpha_{2}}\left(x_{2}\right)\right\rangle_{M+S D}^{U=0} & =\left\langle\tilde{F}_{\alpha_{1}}\left(x_{1}\right) \tilde{F}_{\alpha_{2}}\left(x_{2}\right)\right\rangle_{M+M C S}^{U=0}-i \delta_{\alpha_{1} \alpha_{2}} \frac{\delta\left(x_{1}-x_{2}\right)}{m^{2}} \\
\left\langle\prod_{i=1}^{4} f_{\alpha_{i}}\left(x_{i}\right)\right\rangle_{M+S D}^{U=0} & =\left\langle\prod_{i=1}^{4} \tilde{F}_{\alpha_{i}}\left(x_{i}\right) \cdots\right\rangle_{M+M C S}^{U=0}+\sum_{i \neq j \neq k \neq l} \frac{\delta_{\alpha_{i} \alpha_{j}} \delta_{\alpha_{k} \alpha_{l}}}{2 m^{4}} \delta\left(x_{i}-x_{j}\right) \delta\left(x_{k}-x_{l}\right) \\
& -\sum_{i \neq j \neq k \neq l} i \frac{\delta_{\alpha_{i} \alpha_{j}}}{2 m^{2}} \delta\left(x_{i}-x_{j}\right)\left\langle\tilde{F}_{\alpha_{k}}\left(x_{k}\right) \tilde{F}_{\alpha_{l}}\left(x_{l}\right)\right\rangle_{M+M C S}^{U=0}
\end{aligned}
$$

Similarly, higher point correlation functions will only differ by contact terms. The final result is such that the map $f_{\nu} \leftrightarrow \tilde{F}_{\nu}=\epsilon_{\nu \alpha \beta} \partial^{\alpha} A^{\beta} / m+e J_{\nu}^{(0)} / m^{2}$ holds at quantum level up to contact terms which vanish for non-coinciding points. Formulas (30), (334), (35) ,(39) and (40) are strong indications that the interacting theories $\mathcal{L}_{M+M C S}$ and $\mathcal{L}_{M+S D}$ are equivalent also at quantum level. It must be stressed that the above equivalence has been demonstrated only in the linear coupling case $U\left(\varphi, \varphi^{*}\right)=0$ which coincides with the minimal coupling $\partial_{\nu} \rightarrow \partial_{\nu}-\imath e f_{\nu}$ only for the case of fermions or complex vector fields in a first order formulation as in [23. For the case of charged scalar fields the minimal coupling prescription requires $U\left(\phi, \phi^{*}\right)=2 e^{2} \phi^{*} \phi$. Thus, in order to be able to demonstrate the quantum equivalence we are forced to abandon the minimal coupling for scalar fields. It is tempting to blame the second order formulation of the scalar fields for such problems. We have tried to rewrite them as a first order theory along the lines of [23] with the introduction of auxiliary complex vector fields. However, this has only shifted the problem from the integral over the self-dual field to the integral over the auxiliary vector fields which have also a badly behaved propagator. 


\section{Conclusion}

We have proposed a new master action and proved the on shell equivalence between the self-dual and a Maxwell Chern-Simons type theory non-minimally coupled to $U(1)$ charged matter. The demonstration holds for a rather general coupling to the self-dual field and it does not depend on details of the matter sector.

At the quantum level, we have been able to prove the equivalence between the dual models only in the linear coupling case. It is also in this case that we have a true Maxwell Chern-Simons theory on the dual side. For a general coupling an awkward extra term must be added to the MCS type theory. Although this term is on shell ineffective it gives rise to nonrenormalizable infinities in the quantum theory. The origin of this problem is the fact that the self-dual field behaves like a non-dynamical field (delta function propagator) for short distances. Usually, interaction vertices contain only one line of a non-dynamical field ( linear coupling ), like in the Thirring model, where the quartic interaction can be produced by Gaussian integrating over a non-dynamical vector field linearly coupled with the $U(1)$ fermionic current. If we have double lines of non-dynamical fields in the same vertex, like in $U\left(\varphi, \varphi^{*}\right) f^{\nu} f_{\nu}$, the integration over such fields will in general lead to severe infinities due to loops containing only non-dynamical fields internal lines. In summary, we should avoid quadratic ( and higher ) couplings in the self-dual field.

Finally, the remarks made here might be useful for the coupling with $U(1)$ matter of generalizations of the self dual model, which has been recently investigated in [17, 18, as well as for the nonabelian version of the SD/MCS duality which is under debate in the literature [14]. The nonabelian SD model is a truly interacting theory where the self-dual field has also a bad ultraviolet behavior.

\section{Acknowledgements}

This work was partially supported by CNPq, FAPESP and CAPES, Brazilian research agencies. We thank Alvaro de Souza Dutra and Marcelo Hott for discussions.

\section{References}

[1] S. Coleman, Phys. Rev. D11 (1975) 2088, Ann. Phys. 101 (1976) 239.

[2] S. Mandelstam, Phys. Rev. D 11 (1975) 3026. 
[3] E. Abdalla, M. C. Abdalla and K. D. Rothe, "Non-perturbative methods in two dimensional quantum field theory". World Scientific 1991 - Singapore.

[4] N. Seiberg and E. Witten, Nucl. Phys. B 246 (1994) 19.

[5] P.K. Townsend, K. Pilch and P. van Nieuwenhuizen, Phys. Lett B 136 (1984)38.

[6] S. Deser, R. Jackiw, Phys. Lett. B139 (1984) 371.

[7] E. Fradkin and F. A. Schaposnik, Phys. Lett. B 338 (1994) 253. G. Rossini and F. A. Schaposnik, Phys. Lett. B 338 (1994) 465.

[8] Kei-Ichi Kondo, Progr. Theor. Phys. 94 (1995) 899.

[9] R. Banerjee and E. C. Marino, Mod. Phys. Lett. A14 (1999) 593.

[10] D.G. Barci, L.E. Oxman and S.P. Sorella, Phys. Rev. D 59 (1999) 105012.

[11] D. Dalmazi, A. de Souza Dutra and M. Hott, Phys. Rev. D 67 (2003) 125012.

[12] M. Botta Cantcheff and J. A. Helayel-Neto, Phys. Rev. D 67 (2003) 025016.

[13] R. Banerjee, H.J. Rothe and K. D. Rothe, Phys. Rev. D 52 (1995) 3750.

[14] A. Ilha and C. Wotzasek, Nucl. Phys. B604 (2001) 426, M. Botta Cantcheff, Phys.Lett. B528 (2002) 283-287, D. C. Rodrigues and C. Wotzasek, hep-th/0305141, T.Mariz, R. Menezes, J.R.S. Nascimento, R.F.Ribeiro, C. Wotzasek, het-th/0306265,

[15] M. Gomes, L. C. Malacarne and A. J. da Silva, Phys. Lett. B439 (1998) 137.

[16] M.A. Anacleto, A. Ilha, J.R.S. Nascimento, R.F. Ribeiro and C. Wotzasek, Phys. Lett. B504 (2001) 268.

[17] D. Bazeia, A. Ilha, J.R.S. Nascimento, R.F. Ribeiro and C. Wotzasek, Phys. Lett. B510 (2001) 329.

[18] R. Menezes, J.R.S. Nascimento, R.F. Ribeiro and C. Wotzasek, Phys. Lett. B537 (2002) 321.

[19] D. Bazeia, R. Menezes, J.R. Nascimento, R.F. Ribeiro and C. Wotzasek, Journal of Phys. A36 (2003) 9943.

[20] H. O. Girotti, Int. J. of Mod. Phys. A14 (1999) 2495. 
[21] M.A. Anacleto, J.R.S. Nascimento and R.F. Ribeiro, Phys. Rev. D62 (2000) 067701.

[22] M. Gomes, R. S. Mendes, R.F. Ribeiro and A. J. da Silva. Phys. Rev. D43 (1991) 3516.

[23] C. D. Fosco, V.E. R. Lemes, L. E. Oxman, S. P. Sorella and O. S. Ventura, Annals Phys. 290 (2001) 27. 